Gynäk. Rdsch. 1970;9:159

\title{
Prüfungsfragen für Fachärzte
}

51. Die Implantation der befruchteten Eizelle erfolgt meist:

im Uterushorn

in der Vorderwand des Uterus

in der Hinterwand des Uterus

in der Seitenwand des Uterus

im unteren Uterinsegment

52. Welche Faktoren beeinflussen den Blutstrom durch den interviUösen

Raum?

der Tonus des Uterus

die Kontraktilität der Plazenta

die Bewegung der Zotten

das Herzschlagvolumen

alle diese genannten Faktoren

53. Die Gesamteisenmenge im Körper einer gesunden Frau beträgt:

$2000 \mathrm{mg}$

$3000 \mathrm{mg}$

$4000 \mathrm{mg}$

$5000 \mathrm{mg}$

$6000 \mathrm{mg}$

54. Fehlgeburt - Abortus nennt man die Beendigung der Schwangerschaft vor Ablauf:

von 12 Wochen

von 15 Wochen

von 21 Wochen

von 27 Wochen

55. Die Todesursache bei Fruchtwasserembolie ist:

massive Blutung

Afibrinogenämie

akutes Herzversagen

Hirn-Infarkt

akutes Nierenversagen

56. Wie lange dauert es, bis ein Carcinoma in situ sich zu einem invasiven

Karzinom weiter entwickelt?

10 Jahre

2 Jahre

17 Jahre

unbekannt

7 Jahre

160 
Prüfungsfragen für Fachärzte

57. Mit welcher Häufigkeit ist in der Schwangerschaft mit einem Zervixkarzinom zu rechnen?

$0,2 \%$

$1 \%$

$3 \%$

$3,5 \%$

58. Die häufigsten Befunde am Endometrium bei dysfunktionellen Blutungen sind:

Hyperplasie

Sekretionsphase

Proliferationsphase

alle genannten Befunde

59. Verhalten, wenn bei der Radium-Einlage in das Uteruskavum eine Perforation bemerkt wird:

Fortsetzen und Beenden der Therapie

Abbruch der Therapie und Beobachten für 1-2 Tage

sofortige Laparotomie

Ubergang auf eine andere Therapie besser als Abwarten

Beenden der Therapie und Abwarten für 1-2 Wochen

60. Bei welchem Ovarialtumor ist die Wahrscheinlichkeit einer Malignität am grössten?

Dermoid

serösem Kystadenom

muzinösem Kystadenom

Kystadenofibrom

Brenner-Tumor

Richtige Antworten der Frage $\pi$ 41-50 aus Heft Nr. 1

Frage $41=d$

$=\mathrm{a}$

$=\mathrm{a}$

$=\mathrm{c}$

$=\mathrm{e}$

$=\mathrm{d}$

$=\mathrm{b}$

$=\mathrm{c}$

$=\mathrm{a}$

$=\mathrm{b} 160$

Prüfungsfragen für Fachärzte

57. Mit welcher Häufigkeit ist in der Schwangerschaft mit einem Zervixkarzinom zu rechnen?

$0,2 \%$

$1 \%$

$3 \%$

$3,5 \%$ 
58. Die häufigsten Befunde am Endometrium bei dysfunktionellen Blutungen sind:

Hyperplasie

Sekretionsphase

Proliferationsphase

alle genannten Befunde

59. Verhalten, wenn bei der Radium-Einlage in das Uteruskavum eine

Perforation bemerkt wird:

Fortsetzen und Beenden der Therapie

Abbruch der Therapie und Beobachten für 1-2 Tage

sofortige Laparotomie

Ubergang auf eine andere Therapie besser als Abwarten

Beenden der Therapie und Abwarten für 1-2 Wochen

60. Bei welchem Ovarialtumor ist die Wahrscheinlichkeit einer Malignität am grössten?

Dermoid

serösem Kystadenom

muzinösem Kystadenom

Kystadenofibrom

Brenner-Tumor

Richtige Antworten der Frage $\pi$ 41-50 aus Heft Nr. 1

Frage $41=\mathrm{d}$

$=\mathrm{a}$

$=\mathrm{a}$

$=\mathrm{c}$

$=\mathrm{e}$

$=\mathrm{d}$

$=\mathrm{b}$

$=\mathrm{c}$

$=\mathrm{a}$

$=\mathrm{b}$ 